УДК 519.2:303.732.4

01.00.00 Физико-математические науки

АСИМПТОТИКА КВАНТОВАНИЯ, ВЫБОР
ЧИСЛА ГРАДАЦИЙ В СОЦИОЛОГИЧЕСКИХ
АНКЕТАХ И ДВУХУРОВНЕВАЯ МОДЕЛЬ
УПРАВЛЕНИЯ ЗАПАСАМИ

Орлов Александр Иванович

д.э.н., д.т.н., к.ф.-м.н., профессор

РИНЦ SPIN-код: 4342-4994

Московский государственный технический университет им. Н.Э. Баумана, Россия, 105005,

Москва, 2-я Бауманская ул., 5, prof-orlov@ mail.ru

Рассмотрим один подход к определению шага квантования (группировки) при переходе от непрерывной шкалы к дискретной. Прикладная цель - выбор числа градаций в социологических анкетах. В соответствии с методологией общей теории устойчивости предлагаем выбирать шаг так, чтобы ошибки, порожденные квантованием, были того же порядка, что и ошибки, присущие ответам респондентов (опрашиваемых). При конечном интервале изменения значений измеряемого признака шаг шкалы однозначно определяет число градаций. Оказывается, во многих вопросах закрытого типа достаточно указывать 3 - 6 градаций ответов (подсказок). На основе вероятностной модели доказаны три теоремы о квантовании. Они позволили разработать рекомендации по выбору числа градаций в социологических анкетах. Идея «квантования» имеет применения не только в социологии. Отметим, что применять ее можно не только для выбора числа градаций. Так, весьма интересны два применения идеи «квантования» в теории управления запасами - в двухуровневой модели и в классической модели Вильсона с учетом отклонений от нее (демонстрируется польза «квантования» как способа повышения устойчивости). Для двухуровневой модели управления запасами доказаны три теоремы. Мы отказались от предположения пуассоновости спроса, которое редко выполняется на практике, и получили в общем случае достаточно простые формулы для нахождения оптимальных значений управляющих параметров, попутно исправив ошибки предшественников. В очередной раз видим взаимопроникновение статистических методов, возникших для анализа данных из различных предметных областей, в данном случае, из социологии и логистики. Имеем еще одно доказательство того, что статистические методы единая научно-практическая область, которую нецелесообразно делить по областям применения

Ключевые слова: МАТЕМАТИКА,
UDC 519.2:303.732.4

Physics and mathematical sciences

\section{ASYMPTOTICS OF QUANTIZATION, SELECTION OF THE NUMBER OF GRADATIONS IN THE SOCIOLOGICAL QUESTIONNAIRES AND TWO-LEVEL MODEL OF INVENTORY MANAGEMENT}

Orlov Alexander Ivanovich

Dr.Sci.Econ., Dr.Sci.Tech., Cand.Phys-Math.Sci., professor

Bauman Moscow State Technical University, Moscow, Russia

We consider an approach to the transition from continuous to discrete scale which was defined by means of step of quantization (i.e. interval of grouping). Applied purpose is selecting the number of gradations in sociological questionnaires. In accordance with the methodology of the general stability theory, we offer to choose a step so that the errors, generated by the quantization, were of the same order as the errors inherent in the answers of respondents. At a finite length of interval of the measured value change of the scale this step of quantization uniquely determines the number of gradations. It turns out that for many issues gated it is enough to point $3-6$ answers gradations (hints). On the basis of the probabilistic model we have proved three theorems of quantization. They are allowed to develop recommendations on the choice of the number of gradations in sociological questionnaires. The idea of "quantization" has applications not only in sociology. We have noted, that it can be used not only to select the number of gradations. So, there are two very interesting applications of the idea of "quantization" in inventory management theory - in the two-level model and in the classical Wilson model taking into account deviations from it (shows that "quantization" can use as a way to improve stability). For the two-level inventory management model we proved three theorems. We have abandoned the assumption of Poisson demand, which is rarely carried out in practice, and we give generally fairly simple formulas for finding the optimal values of the control parameters, simultaneously correcting the mistakes of predecessors. Once again we see the interpenetration of statistical methods that have arisen to analyze data from a variety of subject areas, in this case, from sociology and logistics. We have another proof that the statistical methods - single scientificpractical area that is inappropriate to share by areas of applications

Keywords: MATHEMATICS, SOCIOLOGY, 
СОЦИОЛОГИЯ, ЭКОНОМИКА, УПРАВЛЕНИЕ, АНКЕТЫ, ШКАЛЫ, КВАНТОВАНИЕ, УСТОЙЧИВОСТЬ, ВЕРОЯТНОСТНАЯ МОДЕЛЬ, ПРЕДЕЛЬНЫЕ ТЕОРЕМЫ, УПРАВЛЕНИЕ ЗАПАСАМИ, ДВУХУРОВНЕВАЯ МОДЕЛЬ
ECONOMICS, MANAGEMENT, QUESTIONNAIRES, SCALE, QUANTIZATION, STABILITY, PROBABILISTIC MODELS, LIMIT THEOREMS, INVENTORY MANAGEMENT, TWO-LEVEL MODEL

\section{1. Введение}

Наличие погрешностей наблюдений приводит к тому, что результат наблюдения приходится рассматривать не как число, а как объект более сложной природы. «Размытость» реального результата наблюдения можно моделировать различными способами. В классической метрологии погрешность моделируется случайной величиной, математическое ожидание которой называют систематической ошибкой, a соответствующую центрированную случайную величину - случайной ошибкой. В статистике применяют группировку данных [1]. Более общий подход состоит в использовании нечетких чисел [2, 3]. Развиты методы статистики интервальных данных $[4,5]$. Наличие погрешностей наблюдений приводит к существенному изменению свойств статистических процедур. Проанализируем некоторые примеры учета влияния погрешностей, связанные с асимптотикой квантования и ее применением для выбора числа градаций в социологических анкетах и нахождения оптимальных значений управляющих параметров в двухуровневой модели управления запасами.

\section{2. Об учете ошибок в ответах респондентов}

Рассмотрим один подход к определению шага квантования (группировки) при переходе от непрерывной шкалы к дискретной. Прикладная цель - выбор числа градаций в социологических анкетах [6]. В соответствии с методологией общей теории устойчивости [7 - 9] предлагаем выбирать шаг так, чтобы ошибки, порожденные квантованием, были того же порядка, что и ошибки, присущие ответам респондентов 
(опрашиваемых). При конечном интервале изменения значений измеряемого признака шаг шкалы однозначно определяет число градаций. Оказывается, во многих вопросах закрытого типа достаточно указывать 3 6 градаций ответов (подсказок). Этот вполне конкретный вывод следует из теории, развитой в настоящей статье.

Разработаем вероятностную модель, которую будем изучать. Пусть $v$ - истинное значение какого-либо признака. Респондент «измеряет» это значение с какой-то ошибкой, и его мнение отражает число $\xi=v+\alpha$, где $\alpha$ - случайная ошибка, присущая респонденту. Социолог просит респондента назвать не $\xi$, а одну из градаций, включенных в систему баллов, т.е. одно из чисел $k h, k=0, \pm 1, \pm 2, \pm 3, \ldots$, где $h$ - шаг шкалы. Естественно принять модель, согласно которой респондент называет градацию $\eta$, наиболее близкую к его мнению $\xi$. В результате социолог получает от респондента число $\eta=\xi+\beta$, где $\eta \in\{k h, k=0, \pm 1, \pm 2, \pm 3, \ldots\} \quad$ и $-h / 2 \leq \beta<h / 2$. Здесь $\beta$ методическая ошибка, порожденная инструментом социолога - системой баллов.

Очевидно, описанная процедура с ошибками двух типов - ошибкой измерения и ошибкой округления - применима для описания процесса измерения в различных областях, если итог выражается в равномерной шкале с конечным числом градаций. В частности, при оценке качества продукции в баллах, при выводе результата измерения на экран в цифровой форме, и т.д.

Рассмотрим теперь $n$ респондентов, независимо друг от друга отвечающих на вопросы социолога. Респонденту с номером $i$ соответствует истинное значение $v_{i}$ (например, месячный доход или средние затраты времени на просмотр программ телевидения), о которых респондент имеет мнение $\xi_{i}=v_{i}+\alpha_{i}$ (или же: хочет сообщить социологу $\xi_{i}$, a не $\left.v_{i}\right)$. Используя систему баллов с шагом $h$, социолог получает 
$\eta_{i}=\xi_{i}+\beta_{i}$. Предположим, что социолога интересует среднее истинных значений по выборке, т.е.

$$
\bar{v}=\frac{1}{n} \sum_{i=1}^{n} v_{i}
$$

Однако социолог может вычислить лишь

$$
\bar{\eta}=\frac{1}{n} \sum_{i=1}^{n} \eta_{i}=\bar{v}+\bar{\alpha}+\bar{\beta}
$$

где

$$
\bar{\alpha}=\frac{1}{n} \sum_{i=1}^{n} \alpha_{i}, \quad \bar{\beta}=\frac{1}{n} \sum_{i=1}^{n} \beta_{i}
$$

В (1) $\bar{\alpha}$ - ошибка, обусловленная природой респондентов, $\bar{\beta}$ - ошибка, связанная с использованием исследовательского инструмента - системы баллов. Уменьшение шага $h$ (т.е. увеличение числа градаций) уменьшает суммарную ошибку, уменьшая дисперсию $\bar{\beta}$, однако дисперсия $\bar{\alpha}$ при этом не меняется, а потому дисперсия суммарной ошибки всегда больше дисперсии $\bar{\alpha}$. С другой стороны, увеличение числа градаций приводит к возрастанию сложности проведения опроса, к увеличению затрат на социологическое исследование. Поэтому чрезмерное увеличение числа градаций, т.е. чрезмерное уменьшение $h$, нецелесообразно. Согласно «принципу уравнивания погрешностей» в общей схеме устойчивости (см. [7 - 9]) рациональным является такое $h$, при котором ошибки респондентов и ошибки исследовательского инструмента имеют одинаковый порядок. Чтобы вычислить указанные ошибки, необходимо построить вероятностную модель поведения совокупности респондентов.

Будем считать, что $\xi_{i}$ являются независимыми случайными величинами, имеющими математические ожидания и дисперсии, причем

$$
M\left(\xi_{i}\right)=v_{i}, \quad i=1,2, \ldots, n,
$$

т.е. мнение респондента, зависящее от случайных величин, является в среднем правильным, другими словами, не содержит систематической 
ошибки). (Это предположение, разумеется, выполнено не всегда - влияние систематических погрешностей на статистические выводы продемонстрировано в статистике интервальных данных, см., например, $\left[10\right.$, гл.12].) Отметим, что $\beta_{i}$ также являются независимыми случайными величинами (поскольку $\beta_{i}$ - функции от случайных величин $\alpha_{i}$ ). Ниже будет показано, что

$$
\lim _{h \rightarrow 0} h^{-1} M\left(\beta_{i}\right)=0,
$$

кроме того, $\alpha_{i}$ и $\beta_{i}$ при каждом $i$ являются асимптотически независимыми при $h \rightarrow 0$. При малом $h$ и большом $n$ в силу многомерной центральной предельной теоремы и теорем о наследовании сходимости (см., например, [11]) величины $\bar{\alpha}$ и $\bar{\beta}$ можно считать независимыми и нормально распределенными, а потому при указанных условиях случайная величина $\bar{\eta}$ асимптотически нормальна, причем

$$
M(\bar{\eta}) \approx \bar{v}, D(\bar{\eta}) \approx D(\bar{\alpha})+D(\bar{\beta}) .
$$

Условие «однопорядковости ошибок» приводит к рекомендации определять $h$ из соотношения

$$
D(\bar{\alpha})=D(\bar{\beta}) .
$$

В [1] установлено, что

$$
\lim _{h \rightarrow 0} \frac{12 D\left(\beta_{i}\right)}{h^{2}}=1 .
$$

Поэтому $D(\bar{\beta})$ в (2) можно заменить при $h \rightarrow 0$ на $\left(n^{-1} h^{2}\right) / 12$. Если предположить, что ошибки всех респондентов имеют одинаковую дисперсию, т.е. $D\left(\alpha_{i}\right)=\sigma^{2}$, то (2) можно преобразовать к эквивалентному при $h \rightarrow 0$ виду

$$
\frac{\sigma^{2}}{n}=\frac{h^{2}}{12 n}
$$

откуда

$$
h=2 \sqrt{3} \sigma \approx 3,47 \sigma .
$$


Соотношения (3) и (4) можно получить другим путем, исходя из уравнивания вероятностей неправильного упорядочения двух совокупностей (по двум рассматриваемым причинам). Опишем в терминах социологии соответствующую постановку задачи.

Пусть каждый респондент оценивает значения двух признаков. Так, в известных исследованиях В.Н. Шубкина [12] школьники оценивали привлекательность различных профессий. Пусть $v_{i}(1)$ и $v_{i}(2)-$ «истинные значения» рассматриваемых признаков для $i$-го респондента, $\xi_{i}(1)$ и $\xi_{i}(2)-$ то, что $i$-й респондент хотел бы сообщить социологу, т.е. истинные значения, искаженные из-за ошибок респондента; $\eta_{i}(1)$ и $\eta_{i}(2)$ полученные социологом ответы, «привязанные» к градациям шкалы. Можно интерпретировать дисперсии $D\left(\xi_{i}(1)\right)$ и $D\left(\xi_{i}(2)\right)$ как показатели «нечеткости» мнений респондентов (ср. [13]), их изменчивости при повторном опыте через небольшой промежуток времени $[14,15]$.

Пусть $\bar{v}_{1}, \bar{\xi}_{1}, \bar{\eta}_{1}, \bar{\alpha}_{1}, \bar{\beta}_{1}$ - средние арифметические соответствующих показателей для первого признака, а $\overline{\boldsymbol{v}}_{2}, \bar{\xi}_{2}, \bar{\eta}_{2}, \bar{\alpha}_{2}, \bar{\beta}_{2}$ - для второго. Предположим, социолога интересует, что больше - $\bar{v}_{1}$ или $\bar{v}_{2}$. Для ответа на этот вопрос он сравнивает $\bar{\eta}_{1}$ и $\bar{\eta}_{2}$, причем принимает, что $\bar{v}_{1}-\bar{v}_{2}$ имеет тот же знак, что и $\bar{\eta}_{1}-\bar{\eta}_{2}$. Такая процедура может привести к ошибочному заключению о знаке $\bar{v}_{1}-\bar{v}_{2}$. Опять можно выделить ошибки респондента и ошибки исследовательского инструмента. Последние имеют место, если

$$
\bar{\xi}_{1} \neq \bar{\xi}_{2}, \quad\left(\bar{\xi}_{1}-\bar{\xi}_{2}\right)\left(\bar{\eta}_{1}-\bar{\eta}_{2}\right) \leq 0 .
$$

Пусть для определенности $\bar{v}_{1}-\bar{v}_{2}=v>0$. Описанная выше процедура приводит к ошибке, если $\bar{\eta}_{1}-\bar{\eta}_{2} \leq 0$, т.е.

$$
\bar{\alpha}_{2}-\bar{\alpha}_{1}+\bar{\beta}_{2}-\bar{\beta}_{1} \geq v \text {. }
$$

Чтобы найти вероятность осуществления события (5), построим вероятностную модель. Примем все предположения, описанные выше для 
одного признака, и добавим, что оценивание различных признаков проводится независимо. Тогда $\bar{\alpha}_{1}, \bar{\alpha}_{2}, \bar{\beta}_{1}, \bar{\beta}_{2}$ при больших $n$ и малых $h$ можно рассматривать как четыре независимые в совокупности нормальные случайные величины с нулевыми математическими ожиданиями. Тогда $\left(\bar{\alpha}_{2}-\bar{\alpha}_{1}+\bar{\beta}_{2}-\bar{\beta}_{1}\right)$ - нормальная случайная величина с нулевым математическим ожиданием и дисперсией $D\left(\bar{\alpha}_{1}\right)+D\left(\bar{\alpha}_{2}\right)+D\left(\bar{\beta}_{1}\right)+D\left(\bar{\beta}_{2}\right)$, а потому вероятность осуществления события (5) есть

$$
1-\Phi\left(\frac{v}{\sqrt{D\left(\bar{\alpha}_{1}\right)+D\left(\bar{\alpha}_{2}\right)+D\left(\bar{\beta}_{1}\right)+D\left(\bar{\beta}_{2}\right)}}\right),
$$

где $\Phi(z)$ - функция распределения стандартной нормальной случайной величины с математическим ожиданием 0 и дисперсией 1. Условие «однопорядковости» порождающих ошибку причин приводит к равенству

$$
D\left(\bar{\alpha}_{1}\right)+D\left(\bar{\alpha}_{2}\right)=D\left(\bar{\beta}_{1}\right)+D\left(\bar{\beta}_{2}\right) .
$$

В случае $D\left(\bar{\alpha}_{1}\right)=D\left(\bar{\alpha}_{2}\right)$ и $D\left(\bar{\beta}_{1}\right)=D\left(\bar{\beta}_{2}\right)$ условие (6) переходит в (2), а при одинаковости дисперсий респондентов - в (4).

Процесс получения случайной величины $\eta$ по случайной величине $\xi$ естественно назвать «квантованием» или, если угодно, «дискретизацией». Выше описаны способы применения квантования в социологии. Есть и другие возможности его применения, связанные, например, с управлением запасами (в конце настоящей статьи приведены результаты, относящиеся к этой области) или с округлением чисел при вычислениях на ЭВМ.

\section{3. Предельные теоремы о квантовании}

Перейдем к изучению асимптотических свойств квантования при $h \rightarrow 0$. Нам понадобятся вспомогательные утверждения - две леммы.

Лемма 1. Пусть функиии $f: R^{1} \rightarrow R^{1}$ u $g:[0 ;+\infty) \rightarrow[0 ;+\infty)$ таковы, что $|f(x)| \leq g(|x|)$ при всех $x$, функиия g строго убывает, причем существует 


$$
\int_{0}^{+\infty} g(x) d x=B<+\infty
$$

Рассмотрим последовательность точек числовой прямой $a_{k}$ таких, что $a_{k} \in \Delta_{k}=[k h ;(k+1) h), k=0, \pm 1, \pm 2, \ldots, h>0$. Тогда

$$
h \sum_{k}\left|f\left(a_{k}\right)\right|<2 B+2 g(0) h .
$$

Доказательство. Пусть сначала $k \geq 1$. Тогда

$$
\left|f\left(a_{k}\right)\right| \leq g\left(a_{k}\right) \leq g(k h)<g(x)
$$

при всех $x \in \Delta_{k-1}$. Проинтегрировав (7) по $\Delta_{k-1}$, имеем

$$
\left|f\left(a_{k}\right)\right| h<\int_{\Delta_{k-1}} g(x) d x
$$

Из (8) следует оценка

$$
h \sum_{k \geq 1}\left|f\left(a_{k}\right)\right|<\int_{0}^{+\infty} g(x) d x=B .
$$

Аналогично можно получить неравенство

$$
h \sum_{k \leq-2}\left|f\left(a_{k}\right)\right|<B \text {. }
$$

Наконец,

$$
\left|f\left(a_{-1}\right)\right|+\left|f\left(a_{0}\right)\right| \leq 2 g(0) \text {. }
$$

Из (9), (10) и (11) следует требуемое.

Лемма 2. Пусть

а) функиия $f: R^{1} \rightarrow R^{1}$ дважды непрерывно дифференщируема на $R^{l}$;

б) $\int_{-\infty}^{+\infty} f(x) d x=1$;

в) $\lim _{|x| \rightarrow \infty} f(x)=0$;

г) существует строго убывающая интегрируемая функиия $g:[0 ;+\infty) \rightarrow[0 ;+\infty)$ такая, что $\left|f^{\prime \prime}(x)\right| \leq g(|x|)$ при всех $x \in R^{1}$.

Тогда при всех $0 \leq y \leq h$ справедливо неравенство

$$
\left|\sum_{-\infty<k<+\infty} \int_{k h}^{k h+y} f(x) d x-\frac{y}{h}\right|<2,25\left(g(0) h+\int_{0}^{+\infty} g(x) d x\right) h^{2} .
$$

http://ej.kubagro.ru/2016/09/pdf/45.pdf 
Доказательство. Разложим функцию $f(x)$ в точке $a$ по формуле Тейлора с остаточным членом в форме Лагранжа. Пользуясь этим разложением на отрезке $[a ; a+y)$, получим равенство

$$
\int_{a}^{a+y} f(x) d x-\frac{y}{h} \int_{a}^{a+h} f(x) d x=f^{\prime}(a)\left\{\frac{y^{2}}{2}-\frac{y h}{2}\right\}+\left\{K(a, y)-\frac{y}{h} K(a, h)\right\},
$$

где функция $K(a, z)$ такова:

$$
K(a, z)=\int_{a}^{a+z} \frac{(x-a)^{2}}{2} f^{\prime \prime}(\theta a+(1-\theta) x) d x, 0 \leq \theta=\theta(x ; a ; f) \leq 1 .
$$

Полагая в (12) $a=k h, k=0, \pm 1, \pm 2, \ldots$, суммируя по $k$ и пользуясь условием б) настоящей леммы, приходим к разложению

$$
\begin{aligned}
& \sum_{-\infty<k<+\infty} \int_{k h}^{k h+y} f(x) d x-\frac{y}{h}=\left(\frac{y^{2}}{2}-\frac{y h}{2}\right) \sum_{-\infty<k<+\infty} f^{\prime}(k h)+ \\
& +\sum_{-\infty<k<+\infty}\left(K(k h, y)-\frac{y}{h} K(k h, h)\right)
\end{aligned}
$$

Оценим каждую из сумм в правой части (14) по отдельности. Согласно (13) при $0 \leq y \leq h$ имеем

$$
|K(k h, y)| \leq \frac{h^{2}}{2} \max _{x \in \Delta_{k}}\left|f^{\prime \prime}(x)\right| h .
$$

Из (15), леммы 1 и условия г) настоящей леммы следует, что вторая сумма в (14) не превосходит

$$
2 h^{2}\left(g(0) h+\int_{0}^{+\infty} g(x) d x\right) .
$$

С помощью формулы Тейлора и условия в) нетрудно показать, что

$$
h \sum_{-\infty<k<+\infty} f^{\prime}(k h)=-\sum_{-\infty<k<+\infty} L(k h, h),
$$

где

$$
L(a, h)=\int_{a}^{a+h}(x-a) f^{\prime \prime}\left(\theta_{1} a+\left(1-\theta_{1} x\right) d x, 0 \leq \theta_{1}=\theta_{1}(x ; a, f) \leq 1 .\right.
$$

Поскольку

$$
|L(k h, h)| \leq h^{2}\left(\max _{x \in \Delta_{k}}\left|f^{\prime \prime}(x)\right|\right)
$$

http://ej.kubagro.ru/2016/09/pdf/45.pdf 
то с помощью условия г) и леммы 1 заключаем, что

$$
\sum_{-\infty<k<+\infty}|L(k h, h)| \leq h\left(2 h g(0)+2 \int_{0}^{+\infty} g(x) d x\right) .
$$

Из (14), (17), (18) и неравенства

$$
\left|y^{2}-y h\right| \leq \frac{h^{2}}{4}
$$

следует, что первая сумма в (14) не превосходит

$$
0,25\left(\int_{0}^{+\infty} g(x) d x+h g(0)\right) h^{2} .
$$

Из (16) и (19) вытекает заключение леммы 2. Итак, лемма 2 полностью доказана.

Теорема 1. Пусть плотность $f(x)$ случайной величины $\xi$ удовлетворяет условиям леммь 2. Пусть $\eta$ - ближайший $\kappa \xi$ элемент системы баллов $\{k h, k=0, \pm 1, \pm 2, \ldots\}$, m.e. $\eta=\xi+h \alpha_{0}$, где $\eta \in\{k h, k=0, \pm 1$, $\pm 2, \ldots\}$ u $-0,5 \leq \alpha_{0}<0,5$. Тогда $\xi u \alpha_{0}$ асимптотически независимы при $h \rightarrow 0$, m.e.

$$
\limsup _{h \rightarrow 0}\left|P\left\{\xi<x, \alpha_{0}<y\right\}-P\{\xi<x\} P\left\{\alpha_{0}<y\right\}\right|=0 .
$$

Доказательство. Пусть целая часть числа $\frac{x}{h}+0,5$ равна $z$. Имеем

$$
\begin{aligned}
& P\left\{\xi<x, \alpha_{0}<y\right\}=\sum_{-\infty<k \leq z} P\left\{\left(k-\frac{1}{2}\right) h \leq \xi<\left(k+\frac{1}{2}\right) h, \alpha_{0}<y\right\}+ \\
& +P\left\{\left(z-\frac{1}{2}\right) h \leq \xi<\min (x<h y+z h)\right\}
\end{aligned} .
$$

Незначительное изменение доказательства леммы 2 дает, что сумма в формуле (21) есть

$$
\left(\left(y+\frac{1}{2}\right) P\left\{\xi<\left(z-\frac{1}{2}\right) h\right\}+O(h) .\right.
$$

Действительно, 


$$
\sum_{-\infty<k \leq z} P\left\{\left(k-\frac{1}{2}\right) h \leq \xi<\left(k+\frac{1}{2}\right) h, \alpha_{0}<y\right\}=\sum_{-\infty<k \leq z} \int_{(k-0,5) h}^{k h+y h} f(x) d x .
$$

Оценка (16) для суммы в (23) сохраняется. Вместо (17) имеем

$$
h \sum_{-\infty<k \leq z} f^{\prime}\left(\left(k-\frac{1}{2}\right) h\right)=f\left(\left(z-\frac{1}{2}\right) h\right)-\sum_{-\infty<k \leq z} L\left(\left(k-\frac{1}{2}\right) h, h\right)
$$

и соответственно вместо (19) -

$$
f\left(\left(z-\frac{1}{2}\right) h\right) \frac{h}{8}+\frac{1}{4}\left(\int_{0}^{+\infty} g(x) d x+h g(0)\right) h^{2},
$$

откуда и следует (22), причем равномерно по $y$ и $z$. При этом использовалось, что согласно условиям а) и в) леммы 2

$$
\max _{-\infty<x<+\infty} f(x)<+\infty \text {. }
$$

А именно, из (26) следует равномерность по $z$. Кроме того, из (26) вытекает, что

$$
P\left(\left(z-\frac{1}{2}\right) h \leq \xi<\min (x, h y+h z)\right)=O(h),
$$

причем равномерно по $x, y$. С помощью (22) и (27) можно привести (21) к виду

$$
P\left(\xi<x, \alpha_{0}<y\right)=\left(y+\frac{1}{2}\right) P(\xi<x)+O(h)
$$

При этом использовано то, что

$$
P\{\xi<x\}-P\left\{\xi<\left(z-\frac{1}{2}\right) h\right\}=O(h) .
$$

Наконец, из леммы 2 следует, что

$$
\sup _{-0,5 \leq y \leq 0,5}\left|P\left(\alpha_{0}<y\right)-\left(y+\frac{1}{2}\right)\right|=O\left(h^{2}\right) .
$$

Из (28), (29) и отмеченной выше равномерности оценок следует (20), причем можно добавить, что используемый в (20) супремум есть $O(h)$. Теорема 1 доказана. 
Формула (29) представляет самостоятельный интерес: она показывает, что нормированная ошибка округления $\alpha_{0}$ имеет при $h \rightarrow 0$ асимптотически равномерное распределение на отрезке $[-0,5 ;+0,5]$, причем сходимость весьма быстрая. Этот факт будет использован ниже при применении полученных в настоящем разделе результатов в задачах управления запасами (логистике).

теорема 2. Пусть плотность $f(x)$ случайной величины $\xi$ удовлетворяет условиям а) - 2) леммы 2, причем математическое ожидание $М(\xi)$ сущзествует. Тогда для определенной в теореме 1 нормированной очибки округления $\alpha_{0}$ имеем

$$
\lim _{h \rightarrow 0} M\left(\xi \alpha_{0}\right)=0 .
$$

Доказательство. Теорема 1 показывает, что распределение случайного вектора $\left(\xi, \alpha_{0}\right)$ сходится к распределению случайного вектора $(\xi, \delta)$ при $h \rightarrow 0$, где $\delta$ - независимая от $\xi$ случайная величина с равномерным распределением на отрезке $[-0,5 ;+0,5]$.

Отсюда следует, что при любом фиксированном $N$

$$
\lim _{h \rightarrow 0} M\left(\xi_{N} \alpha_{0}\right)=M\left(\xi_{N} \delta\right),
$$

где

$$
\xi_{N}=\left\{\begin{array}{l}
\xi,|\xi|<N \\
N,|\xi| \geq N
\end{array}\right.
$$

Для доказательства достаточно сослаться на двумерный аналог утверждения, известного как первая теорема Хелли [16, с.344-346], вторая теорема Хелли [17, с.174-175], лемма Хелли - Брея [18, с.193-194] (см. также [10, раздел 7.3] и [19]).

Из независимости $\xi$ и $\delta$ следует, что $\xi_{N}$ и $\delta$ также независимы. Поскольку $M(\delta)=0$, то $M\left(\xi_{N} \delta\right)=0$.

Для завершения доказательства достаточно показать, что 


$$
\lim _{h \rightarrow 0, N \rightarrow+\infty} M\left\{\left(\xi-\xi_{N}\right) \alpha_{0}\right\}=0 .
$$

Действительно, поскольку $\left|\eta \alpha_{0}\right| \leq \frac{1}{2}|\eta|$ при любом $\eta$, то

$$
M\left\{\left(\xi-\xi_{N}\right) \alpha_{0}\right\} \leq \frac{1}{2} M\left(\left|\xi-\xi_{N}\right|\right)=\frac{1}{2} \int_{|x| \geq N}(|x|-N) d P(\xi<x) \leq \frac{1}{2} \int_{|x| \geq N}|x| d P(\xi<x)
$$

Остается заметить что последний член цепочки неравенств (34) стремится к 0 при $N \rightarrow+\infty$ в силу существования математического ожидания $M(\xi)$. Теорема 2 доказана.

Теорема 3. Пусть выполнены условия теоремы 2 и, кроме того, дисперсия $D(\xi) \neq 0$ существует. Тогда при $h \rightarrow 0$ коэффищиент коррелящии между $\xi$ и $\alpha_{0}$ стремится $\kappa 0$.

Доказательство. В силу теоремы 1 (формула (29)) и цитированного в теореме 2 двумерного аналога теоремы Хелли

$$
\lim _{h \rightarrow 0} M\left(\alpha_{0}\right)=0, \lim _{h \rightarrow 0} D\left(\alpha_{0}\right)=\frac{1}{12}=D(\delta) .
$$

В силу теоремы 2

$$
\lim _{h \rightarrow 0} M\left(\xi \alpha_{0}\right)=0 .
$$

Из (35) и (36) следует, что

$$
\lim _{h \rightarrow 0} M\left\{(\xi-M(\xi))\left(\alpha_{0}-M\left(\alpha_{0}\right)\right)\right\}=0 .
$$

Для завершения доказательства теоремы 3 достаточно использовать (37) и еще раз сослаться на второе соотношение в (35) и на то, что $D(\xi)$ существует и не равна 0 .

Доказаны все утверждения, необходимые для обоснования проведенных в начале статьи рассуждений, с помощью которых был получены формулы (3) и (4). Нет необходимости подробно расписывать применение многомерной центральной теоремы и теорем о наследовании сходимости [11] к векторам $(\bar{\alpha}, \bar{\beta})$ и $\left(\bar{\alpha}_{1}, \bar{\alpha}_{2}, \bar{\beta}_{1}, \bar{\beta}_{2}\right)$ для получения этих формул. 


\section{4. Рекомендации по выбору числа градаций в социологических}

\section{анкетах}

Пусть от респондента требуется оценить интенсивность какого-либо качества в процентах (т.е. рассматриваемые величины меняются в интервале от 0\% до 100\%). С помощью формулы (4) можно найти требуемое число градаций $k$. А именно,

$$
k \approx \frac{100}{h} \approx \frac{100}{3,47 \sigma}=\frac{28,8}{\sigma} .
$$

Поскольку $k$ - натуральное число, можно рекомендовать к практическому использованию формулу

$$
k=\left[\frac{28,8}{\sigma}\right]+1,
$$

где квадратными скобками обозначена операция взятия целой части числа. Так, при $\sigma=10(\%)$ в соответствии с (38) $k=3$, при $\sigma=5$ (\%) соответственно $k=6$. Серединными токами интервалов являются $17 \%$, $50 \%, 83 \%$ в первом случае и $8,3 \%, 25 \%$ и т.д. - во втором.

Дисперсию ошибки респондента можно оценить при пилотаже (пробном исследовании) или найти иными способами, имеющимися в многочисленных работах по методике социологических опросов [14]. Для многих социологических данных [15] среднее квадратическое отклонение мнения респондента $\sigma$ имеет порядок 5-10\% и более Аналогичный порядок точности для экономико-статистических данных приводит и Оскар Моргенштерн [20].

Наши результаты показывают, что во многих социологических анкетах целесообразно использовать не более 3 - 6 градаций. Разумеется, эти формально-математические аргументы не могут служить единственным основанием при выборе числа градаций. Какова же их польза? Они могут служить ориентиром и, что по нашему мнению более 
важно, обосновывают корректность эмпирически применяемых мощностей множества градаций (в данном случае мощность конечного множества это число его элементов). По данным [21], массивы данных в социологии на $92 \%$ состоят из значений качественных признаков, что и объясняет больше значение статистики нечисловых данных для социологических исследований. Присоединяясь в целом к высказанному в этой работе [21] пожеланию шире использовать в социологии многомерный статистический анализ и другие методы, основанные на признаках, измеренных в количественных шкалах (интервалов, отношений и др.), необходимо подчеркнуть, что квантование шкалы является эмпирически найденным приемом, повышающим обоснованность выводов статистического анализа социологических данных путем повышения устойчивости к ошибкам респондентов. Выше дано теоретическое обоснование этому эмпирическому приему.

Первоначальный вариант теории асимптотического квантования был опубликован в конце 70-х годов (см. прежде всего [6], а также [7, раздел 2.6]). Они использовались, например, в [22]. Их экспериментальную проверку провел Ф.Н. Ильясов [23], в результате от 13 градаций в исходной шкале перешел к шкале с 6 градациями.

Есть и иные способы определения необходимого числа градаций [24].

\section{5. Применения в управлении запасами}

Идея «квантования» имеет применения не только в социологии. Отметим, что применять ее можно не только для выбора числа градаций. Так, весьма интересны два применения идеи «квантования» в теории управления запасами - в двухуровневой модели (полезны теоремы 1 - 3) и в классической модели Вильсона [25] с учетом отклонений от нее (демонстрируется польза «квантования» как способа повышения 
устойчивости). В очередной раз видим взаимопроникновение статистических методов, возникших для анализа данных из различных предметных областей, в данном случае, из социологии и логистики. Имеем еще одно доказательство того, что статистические методы - единая научно-практическая область, которую нецелесообразно делить по областям применения.

Введение в теорию управления запасами дано, например, в [26, разд. 8.4]. В математических терминах двухуровневая однопродуктовая модель работы склада формулируется следующим образом.

Пусть $\quad\left\{\xi_{n}, n=1,2, \ldots\right\}-\quad$ последовательность $\quad$ неотрицательных независимых одинаково распределенных случайных величин (последовательность величин заявок со стороны потребителей). Пусть $\tau(t)$ - неубывающий кусочно-постоянный случайный процесс на $[0 ;+\infty)$, независимый от последовательности $\left\{\xi_{n}\right\}$ и имеющий скачки величиной 1 в точках $\tau_{1}<\tau_{2}<\ldots<\tau_{k}<\ldots$, причем $\tau(0)=0$ (процесс $\tau(t)$ описывает моменты возникновения заявок со стороны потребителей). Положим

$$
s(t)=\xi_{1}+\xi_{2}+\ldots+\xi_{\tau(t)}, t \geq 0
$$

(величина $s(t)$ показывает накопленный спрос за время $t$ ). Пусть $R<0$ и $R+Q>0$ - произвольные числа (уровни, упоминаемые в названии модели). Пусть

$$
y(t)=\left[\frac{s(t)+R}{Q}\right] Q+Q-s(t),
$$

где квадратными скобками обозначена целая часть числа. Здесь $y(t)$ уровень запаса продукта на складе. Предполагается, что продукт отпускается в соответствии с заявками потребителей. Когда уровень запаса на складе опускается до граничного значения $R<0$, мгновенно поступает новая партия продукта величиной $Q$. Если уровень запаса на складе при удовлетворении очередной заявки становится меньше 0, но остается 
больше $R<0$, то фиксируется образование дефицита. Рассматриваемая заявка (точнее, ее необслуженная часть) и возникающие в следующие моменты заявки накапливаются вплоть до того момента, когда суммарный дефицит достигнет граничного уровня $R$, a затем мгновенно удовлетворяются после прихода очередной поставки величиной $Q$. Таким образом,

$$
R<y(t) \leq R+Q
$$

при всех $t$.

Пусть $c_{1}, c_{2}, c_{3}$ - положительные константы, $T>0$. Введем функционал

$$
w(T)=\frac{1}{T}\left\{c_{1} \int_{0}^{T} y^{+}(t) d t+c_{2} \int_{0}^{T} y^{-}(t) d t+c_{3}\left[\frac{s(T)+R}{Q}\right]\right\},
$$

где $y^{+}=\max (y, 0)$ и $y^{-}=y^{+}-y$. Требуется минимизировать $M(w(T))$, выбрав соответствующие $R$ и $Q$.

В логистических терминах $c_{1}$ - расходы, связанные с хранением единицы продукта в течение единицы времени, $c_{2}$ - потери, порожденные дефицитом (отсутствием на складе) единицы продукции в течение единицы времени, $c_{3}$ - расходы на доставку партии продукта на склад, $w(T)$ - средние (на единицу времени) издержки на функционирование склада (точнее, складской подсистемы, связанной со снабжением потребителей рассматриваемым продуктом). Сформулированная модель рассматривалась рядом авторов [27 - 32] при различных предположениях. $\mathrm{y}$ них же можно найти экономическую интерпретацию и развернутое обсуждение рассматриваемых математических объектов и констант (см. также [26, разд. 8.4]).

Будем изучать асимптотику при $T \rightarrow \infty$. Сначала найдем предельное распределение $y(t)$. 
Теорема 4. Пусть случайная величина $\xi_{1}$ имеет первые пять моментов $и$ интегрируемую характеристическую функиию, при некотором $C$

$$
P(\tau(t)=0)<C M\left(\frac{1}{\tau(t)+1}\right)
$$

при всех $t \geq 0$. Тогда существует константа D такая, что

$$
\left|P(y(t) \leq y)-\frac{y-R}{Q}\right|<D M\left(\frac{1}{\tau(t)+1}\right)
$$

при всех $y, t$ таких, что $R \leq y \leq R+Q u t \geq 0$.

Доказательство. Нетрудно показать, что

$$
P(y(t) \leq y)=\sum_{n=0}^{+\infty} P(n Q-y \leq s(t)<n Q-R) .
$$

Нам окажется полезным вытекающее из (39) представление

$$
P(y(t) \leq y)=\sum_{k=0}^{+\infty} A(k, y) P(\tau(t)=k),
$$

где

$$
A(k, y)=\sum_{n=0}^{+\infty} P(n Q-y \leq s(t)<n Q-R \mid \tau(t)=k) .
$$

При $k \geq 1$ положим

$$
T_{k}=\frac{\xi_{1}+\xi_{2}+\ldots+\xi_{k}-k M\left(\xi_{1}\right)}{\sqrt{k} \sqrt{D\left(\xi_{1}\right)}}
$$

Тогда

$$
A(k, y)=\sum_{n=0}^{+\infty} P\left(\frac{n Q-y-k M\left(\xi_{1}\right)}{\sqrt{k} \sqrt{D\left(\xi_{1}\right)}} \leq T_{k}<\frac{n Q-R-k M\left(\xi_{1}\right)}{\sqrt{k} \sqrt{D\left(\xi_{1}\right)}}\right) .
$$

Начнем с оценки $A(k, y)$.

Лемма 3. В условиях теоремь 4 существует константа $d_{1}$ такая, что при всех у таких, что $R \leq y \leq R+Q u k=1,2, \ldots$, выполнено неравенство

$$
\left|A(k, y)-\frac{y-R}{Q}\right|<\frac{d_{1}}{k}
$$


Доказательство. Пусть $f_{k}(x)$ - плотность случайной величины $T_{k}$, существующая в силу условий теоремы 4. Из них же в соответствии с теоремой 2 из [33, с.611] вытекает, что

$$
f_{k}(x)-g_{k}(x)=\gamma_{k}(x)=O\left(k^{-3 / 2}\right)
$$

равномерно по $x$. Здесь

$$
g_{k}(x)=\eta(x)\left(1+\frac{\alpha}{\sqrt{k}}\left(x^{3}-3 x\right)+\frac{\beta}{k}\left(x^{4}-6 x^{2}+3\right)\right),
$$

где $\eta(x)$ - плотность стандартного нормального распределения с математическим ожиданием 0 и дисперсией $1, \alpha$ и $\beta$ - константы, зависящие от моментов случайной величины $\xi_{1}$.

Для $A(k, y)$ справедливо представление

$$
A(k, y)=\sum_{n=0}^{+\infty} \int_{a(n)}^{a(n)+b} g_{k}(x) d x+\sum_{n=0}^{+\infty} \int_{a(n)}^{a(n)+b} \gamma_{k}(x) d x,
$$

где

$$
a(n)=n \frac{Q}{\sqrt{k} \sqrt{D\left(\xi_{1}\right)}}-\frac{y+k M\left(\xi_{1}\right)}{\sqrt{k} \sqrt{D\left(\xi_{1}\right)}}, \quad b=\frac{y-R}{\sqrt{k} \sqrt{D\left(\xi_{1}\right)}} .
$$

К первому слагаемому в (43) применим лемму 2, второе слагаемое оценим с помощью (41).

Пусть $m$ - такое целое число, что $a(m) \leq 0$, но $a(m+1)>0$. Легко проверить, что для функции $f(x)=g_{k}(x-a(m))$ выполнены все условия леммы 2. Полагая (слева - обозначения леммы 2, справа - обозначения из (44))

$$
h=\frac{Q}{\sqrt{k} \sqrt{D\left(\xi_{1}\right)}}, y=b,
$$

заключаем, что при некоторой константе $d_{2}$, зависящей, как видно из (42) и (44), лишь от $Q$ и моментов $\xi_{1}$, и произвольном $k$

$$
\left|\sum_{-\infty<n<+\infty} \int_{a(n)}^{a(n)+b} g_{k}(x) d x-\frac{y-R}{Q}\right|<\frac{d_{2}}{k} .
$$

Отметим теперь, что 


$$
\left|\sum_{n<0} \int_{a(n)}^{a(n)+b} g_{k}(x) d x\right| \leq \int_{-\infty}^{c(k)}\left|g_{k}(x)\right| d x
$$

где

$$
c(k)=-\sqrt{k} \frac{M\left(\xi_{1}\right)}{\sqrt{D\left(\xi_{1}\right)}}-\frac{y}{\sqrt{k} \sqrt{D\left(\xi_{1}\right)}} .
$$

Из (42) нетрудно вывести, что

$$
\int_{-\infty}^{c(k)}\left|g_{k}(x)\right| d x=O\left(\frac{1}{k}\right)
$$

Из (45) и (46) следует оценка

$$
\left|\sum_{n=0}^{+\infty} \int_{a(n)}^{a(n)+b} g_{k}(x) d x-\frac{y-R}{Q}\right|=O\left(\frac{1}{k}\right) .
$$

Займемся теперь второй суммой в (43). Положим

$$
N_{1}=\min \{n: a(n)>-\sqrt{k}\}, N_{2}=\max \{n: a(n)+b<\sqrt{k}\} .
$$

Тогда в силу (41)

$$
\left|\sum_{N_{1} \leq n \leq N_{2}} \int_{a(n)}^{a(n)+b} \gamma_{k}(x) d x \leq \int_{-\sqrt{k}}^{\sqrt{k}}\right| \gamma_{k}(x)|d x|=O\left(\frac{1}{k}\right) .
$$

Справедливо неравенство

$$
\left|\sum_{n=0}^{N_{1}-1 a(n)+b} \int_{a(n)} \gamma_{k}(x) d x+\sum_{n=N_{2}+1}^{+\infty} \int_{a(n)}^{a(n)+b} \gamma_{k}(x) d x \leq \int_{B(k)} f_{k}(x) d x+\int_{B(k)}\right| g_{k}(x)|d x|,
$$

где $B(k)=(-\infty ;-\sqrt{k}+b) \bigcup(\sqrt{k}-b ;+\infty)$. Первый интеграл в правой части (49) равен $P\left\{\left|T_{k}\right| \geq \sqrt{k}-b\right\}$. С помощью неравенства Чебышева и (44) заключаем, чTO

$$
P\left\{\left|T_{k}\right| \geq \sqrt{k}-b\right\} \leq \frac{1}{(\sqrt{k}-b)^{2}}=O\left(\frac{1}{k}\right) .
$$

Из явного вида $g_{k}(x)$, по аналогии с (46), заключаем, что второй интеграл в правой части (49) также есть $O\left(\frac{1}{k}\right)$. Из (48), (49), (50) и последнего утверждения следует, что 


$$
\sum_{n=0}^{+\infty} \int_{a(n)}^{a(n)+b} \gamma_{k}(x) d x=O\left(\frac{1}{k}\right)
$$

Из (47) и (51) вытекает заключение леммы 3, поскольку, как нетрудно проверить, просматривая проведенные выше рассуждения, все оценки являются равномерными по $y$. Лемма 3 полностью доказана.

Продолжение доказательства теоремы 4. Из (40) и заключения леммы 3 следует, что

$$
P(y(t) \leq y)=\frac{y-R}{Q}+P(\tau(t)=0)\left(A(0, y)-\frac{y-R}{Q}\right)+d_{1} \sum_{k \geq 1} \frac{\theta_{k}}{k} P(\tau(t)=k),
$$

где $\left|\theta_{k}\right|<1, k=1,2, \ldots$ Поскольку

$$
\left|\sum_{k \geq 1} \frac{\theta_{k}}{k} P(\tau(t)=k)\right| \leq \sum_{k \geq 1} \frac{2}{k+1} P(\tau(t)=k) \leq 2 M\left(\frac{1}{\tau(t)+1}\right),
$$

то заключение теоремы 4 справедливо при $D=2 d_{1}+C$. Теорема 4 доказана.

Теорема 5. В условиях теоремь 4 и при $M(\tau(T))<+\infty$ имеем для минимизируемого функиионала

$$
M(w(T))=H(R, Q)+\delta_{T}(R, Q),
$$

где

$$
H(R, Q)=\left(c_{1}+c_{2}\right) \frac{R^{2}}{2 Q}+c_{1} R+\frac{c_{1} Q}{2}+\frac{c_{3} M(\tau(T)) M\left(\xi_{1}\right)}{Q T},
$$

причем существуют константы $d_{3} u d_{4}$ такие, что

$$
\left|\delta_{T}(R, Q)\right| \leq \frac{d_{3}}{T}+\frac{d_{4}}{T} \int_{0}^{T} M\left(\frac{1}{\tau(t)+1}\right) d t .
$$

Доказательство. Поскольку накопленный спрос есть сумма случайного числа случайных слагаемых, то по тождеству Вальда $M(s(T))=M\left(\xi_{1}\right) M(\tau(T))$. Следовательно, для математического ожидания третьего слагаемого средних издержек $w(T)$ имеем

$$
M\left(c_{3}\left[\frac{s(T)+R}{Q}\right]\right)=\frac{c_{3} M(\tau(T)) M\left(\xi_{1}\right)}{Q T}+\frac{c_{3}}{T} \omega,
$$


где

$$
\frac{R}{Q}-1<\omega \leq \frac{R}{Q}
$$

При изучении математического ожидания первого слагаемого средних издержек $w(T)$ полезно проинтегрировать по частям:

$$
M\left(y^{+}(t)\right)=\left.y P(y(t) \leq y)\right|_{0} ^{R+Q}-\int_{0}^{R+Q} P(y(t) \leq y) d y .
$$

Из (56) и заключения теоремы 4 следует, что

$$
M\left(y^{+}(t)\right)=R+\frac{Q}{2}+\frac{R^{2}}{2 Q}+\lambda
$$

где

$$
|\lambda| \leq(R+Q) D M\left(\frac{1}{\tau(t)+1}\right)
$$

Аналогично

$$
\text { получаем }
$$

асимптотическое

выражение

для математического ожидания второго слагаемого в формуле для $w(T)$ :

$$
M\left(y^{-}(t)\right)=\frac{R^{2}}{2 Q}+\lambda^{\prime}
$$

где

$$
\left|\lambda^{\prime}\right| \leq|R| D M\left(\frac{1}{\tau(t)+1}\right)
$$

Из (54) - (60) следует утверждение теоремы 5, при этом можно считать, что в (53)

$$
d_{3}=c_{3}\left(\frac{|R|}{Q}+1\right), d_{4}=c_{1}(R+Q) D+c_{2}|R| D .
$$

Теорема 5 доказана.

Нетрудно показать, что $H(R, Q)$ из теоремы 5 (см. (52)) достигает минимума по области $\{(R, Q): Q>0\}$ в точке $\left(R_{0}, Q_{0}\right)$, где

$$
R_{0}=-\sqrt{\frac{2 c_{1} c_{3} M\left(\xi_{1}\right) M(\tau(T))}{\left(c_{1}+c_{2}\right) c_{2} T}}, \quad Q_{0}=\sqrt{\frac{2\left(c_{1}+c_{2}\right) c_{3} M\left(\xi_{1}\right) M(\tau(T))}{c_{1} c_{2} T}} .
$$

Теорема 6. Пусть выполнены условия теоремы 5 и, кроме того, 


$$
\varlimsup_{T \rightarrow+\infty} \frac{M(\tau(T))}{T}<+\infty .
$$

Предположим, что минимум $M(w(T))$ достигается, причем в точке $\left(R_{T}\right.$, $Q_{T}$ ). Тогда существует константа $d_{5}$ такая, что при всех $T$

$$
\left|R_{T}-R_{0}\right|+\left|Q_{T}-Q_{0}\right|<d_{5} \sqrt{\frac{1}{T}+\frac{1}{T} \int_{0}^{T} M\left(\frac{1}{\tau(T)+1}\right) d t .}
$$

Доказательство. Нетрудно показать, что в силу (63) минимум $M(w(T))$ не может достигаться вне некоторого компакта. Далее, анализируя доказательства теорем 4 и 5, можно убедиться, что полученные в них оценки являются равномерными по произвольному компакту в области $\{(R, Q): Q>0\}$. Поскольку

$$
H\left(R_{T}, Q_{T}\right) \leq H\left(R_{0}, Q_{0}\right)+\delta_{T}\left(R_{0}, Q_{0}\right)-\delta_{T}\left(R_{T}, Q_{T}\right)=H\left(R_{0}, Q_{0}\right)+\varepsilon,
$$

то $\left(R_{T}, Q_{T}\right)$ лежит в области $\left\{(R, Q): H(R, Q) \leq H\left(R_{0}, Q_{0}\right)+\mathcal{E}\right\}$. Эта область является эллипсом, и, как легко видеть [34],

$$
R_{T}-R_{0}=O(\sqrt{\varepsilon}), Q_{T}-Q_{0}=O(\sqrt{\varepsilon}) .
$$

Из (53), (61) и (64) следует требуемое. Теорема 6 доказана.

Следствие. Пусть выполнены условия теоремы 6 и, кроме того,

$$
\lim _{T \rightarrow+\infty} \frac{1}{T} \int_{0}^{T} M\left(\frac{1}{\tau(t)+1}\right) d t=0
$$

Тогда

$$
\lim _{T \rightarrow+\infty}\left(\left|R_{T}-R_{0}\right|+\left|Q_{T}-Q_{0}\right|\right)=0
$$

и

$$
\lim _{T \rightarrow+\infty}\left(M\left(w\left(t, R_{T}, Q_{T}\right)\right)-M\left(w\left(t, R_{0}, Q_{0}\right)\right)\right)=0,
$$

где величину $M\left(w\left(T, R_{T}, Q_{T}\right)\right)$ рассчитывают по формулам для математического ожидания средних издержек работы склада $M(w(T))$ при параметрах плана поставок $R=R_{T}, Q=Q_{T}$, а величину $M\left(w\left(T, R_{0}, Q_{0}\right)\right)-$ по тем же формулам при $R=R_{0}, Q=Q_{0}$. 
Таким образом, следствие указывает условия, при которых при большом горизонте планирования $T$ возможно использовать асимптотически оптимальный план поставок с параметрами $R=R_{0}, Q=Q_{0}$, заданными формулами (62).

Отметим, что для справедливости соотношения (65) необходимо и достаточно, чтобы

$$
\lim _{t \rightarrow+\infty} M\left(\frac{1}{\tau(t)+1}\right)=0,
$$

но недостаточно выполнения условия

$$
\lim _{t \rightarrow+\infty} M(\tau(t))=+\infty .
$$

В работах $[27,31]$ рассматривался пуассоновский поток $\tau(t)$ заявок от потребителей. Тогда накопленный спрос $s(t)$ асимптотически (при $t \rightarrow+\infty)$ нормален. В этих работах предлагалось вероятности в правой части (39) заменить на предельные и полученным рядом аппроксимировать функцию распределения $P(y(t) \leq y)$ при больших $t$. Чтобы сделать расчеты возможными, т.е. из эвристических побуждений, функция Лапласа в этом ряду заменялась [27] на ступенчатую функцию с единственным скачком в 0 единичной высоты. С помощью результатов настоящей статьи нетрудно подсчитать, что такая замена приводит к появлению лишних членов

$$
2 c_{1} \frac{v}{\mu}(R+Q)+2 c_{2} \frac{v}{\mu} R
$$

в аналогичном $H(R, Q)$ выражении (обозначения [27]). Таким образом, мы отказались от предположения пуассоновости, которое редко выполняется на практике, и получили в общем случае достаточно простые формулы, попутно исправив ошибки предшественников.

Отказ от пуассоновости спроса впервые осуществлен нами в [28]. Дальнейшая работа проводилась совместно с А.В. Воскресенским [34, 35]. Настоящее изложение развивает наброски работы [36], в которой предшествующие результаты были усилены. 
Классическая модель Вильсона управления запасами разобрана в [26, разд. 8.4]. Там показано, что квантование используемых экономических показателей позволяет исключить расхождение между собой методик, разработанных различными коллективами специалистов, но при этом обеспечивает сокращение издержек складского хозяйства не менее чем в 2 paза.

Место задачи о выборе числе градаций в социологических анкетах среди других математических задач социологии обсуждается в [37].

\section{Литература}

1. Орлов А.И. Статистическое оценивание для сгруппированных данных // Политематический сетевой электронный научный журнал Кубанского государственного аграрного университета. 2014. № 98. С. 1-13.

2. Орлов А.И. Теория нечетких множеств - часть теории вероятностей // Политематический сетевой электронный научный журнал Кубанского государственного аграрного университета. 2013. № 92. С. 51-60.

3. Орлов А.И. Статистика нечетких данных // Политематический сетевой электронный научный журнал Кубанского государственного аграрного университета. 2016. № 119. С. 75-91.

4. Орлов А.И. Основные идеи статистики интервальных данных // Политематический сетевой электронный научный журнал Кубанского государственного аграрного университета. 2013. № 94. С. 55-70.

5. Орлов А.И., Луценко Е.В. Системная нечеткая интервальная математика. Монография (научное издание). - Краснодар, КубГАУ. 2014. - 600 с.

6. Орлов А.И. Асимптотика квантования и выбор числа градаций в социологических анкетах // В сб.: Математические методы и модели в социологии. М.: ИСИ АН СССР, 1977. - С. $42-55$.

7. Орлов А.И. Устойчивость в социально-экономических моделях. - М.: Наука, 1979. - 296 с.

8. Орлов А.И. Устойчивые экономико-математические методы и модели. Saarbrücken (Germany), Lambert Academic Publishing, 2011. - 436 c.

9. Орлов А.И. Новый подход к изучению устойчивости выводов в математических моделях // Политематический сетевой электронный научный журнал Кубанского государственного аграрного университета. 2014. № 100. С. 146-176.

10. Орлов А.И. Прикладная статистика. - М.: Экзамен, 2006. - 672 с.

11. Орлов А.И. Теоретические инструменты статистических методов // Политематический сетевой электронный научный журнал Кубанского государственного аграрного университета. 2014. № 101. С. 253-274.

12. Шубкин В.Н. Социологические опыты. - М.: Мысль, 1970. - 256 с.

13. Шошин П.Б. Размытые числа как средство описания субъективных величин // Статистические методы анализа экспертных оценок. - М.: Наука, 1977. - С. 234-250. 
14. Саганенко Г.И. Надежность результатов социологического исследования. - Л.: Наука, 1983. - 180 с.

15. Шляпентох В.Э. Проблемы достоверности статистической информации в социологических исследованиях. - М.: Статистика, 1973. - 144 с.

16. Колмогоров А.Н., Фомин С.В. Элементы теории функций и функционального анализа. Учебник. - М.: Наука, 1972. - 496 с.

17. Гнеденко Б.В. Курс теории вероятностей: Учебник. 7-е изд., исправл. М.: Эдиториал УРСС, 2001. 320 с.

18. Лоэв М. Теория вероятностей. - М.: ИЛ, 1962. - 720 с.

19. Орлов А.И. Предельная теория непараметрических статистик // Политематический сетевой электронный научный журнал Кубанского государственного аграрного университета. 2014. № 100. С. 31-52.

20. Моргенштерн О. О точности экономико-статистических наблюдений. М.: Статистика, 1968. - 396 с.

21. Титма М.Х., Тоодинг Л.М. Математические методы в арсенале социолога. / Социологические исследования. 1986. №4. С.123-128.

22. Глотов В.А., Павельева В.В. Векторная стратификация. - М.: Наука, 1984. $-94 \mathrm{c}$.

23. Ильясов Ф.Н. Экспериментальное обоснование количества делений шкалы. / Социологические исследования. 1984. №4. С.113-116.

24. Литвак Б.Г. О выборе делений шкалы. - В сб.: Статистические методы анализа экспертных оценок. - М.: Наука, 1977. - С.228-234.

25. Орлов А.И. Оптимальный план управления запасами нельзя найти на основе формулы квадратного корня // Политематический сетевой электронный научный журнал Кубанского государственного аграрного университета. 2015. № 106. C. 270-300.

26. Орлов А.И. Организационно-экономическое моделирование : учебник : в 3 ч. Ч.3. Статистические методы анализа данных. - М.: Изд-во МГТУ им. Н.Э. Баумана, 2012. $-624 \mathrm{c}$.

27. Лавренченко А.С. Решение задач оптимального управления запасами при нестационарном пуассоновском потоке требований. // Сб. научных трудов / Московский авиационный институт. - 1974. - Вып.306. - С. 62-72.

28. Орлов А.И. Отказ от пуассоновости спроса в одной модели управления запасами // Исследования по вероятностно-статистическому моделированию реальных систем. - М.: Изд-во ЦЭМИ АН СССР, 1977. - С. 93-96.

29. Ротарь Г.В. Об одной задаче управления резервами // Экономика и математические методы. 1976. Т.12. №4. С. 733-739.

30. Ротарь Г.В. Одна задача об управлении резервами // Теория вероятностей и ее применения. 1972. Т.XVII. Вып.3. С.597-599.

31. Саульев В.К., Лавренченко А.С. Математическая теория оптимального управления запасами. - М.: МАИ, 1974. - 188 с.

32. Хедли Дж., Уайтин Т. Анализ систем управления запасами. - М.: Наука, 1969. $-512 \mathrm{c}$.

33. Феллер В. Введение в теорию вероятностей и её приложения: Том 2. - М.: Мир, 1984. - 738 с.

34. Воскресенский А.В. Принцип инвариантности в одной модели управления запасами / Дипл. работа (руководитель А.И. Орлов). - М.: Мех.-мат. ф-т МГУ им. М.В. Ломоносова, 1977. 
35. Орлов А.И., Воскресенский А.В. Принцип инвариантности в одной модели управления запасами // Прикладной многомерный статистический анализ. Ученые записки по статистике, т.33. - М.: Наука, 1978. - С.307-311.

36. Орлов А.И. Горизонтная устойчивость двухуровневой модели управления запасами // Многомерный статистический анализ (математическое обеспечение). - М.: Изд-во ЦЭМИ АН СССР, 1979. - С.187-199.

37. Орлов А.И. Математические методы в социологии за сорок пять лет // Политематический сетевой электронный научный журнал Кубанского государственного аграрного университета. 2016. № 117. С. 91-119.

\section{References}

1. Orlov A.I. Statisticheskoe ocenivanie dlja sgruppirovannyh dannyh // Politematicheskij setevoj jelektronnyj nauchnyj zhurnal Kubanskogo gosudarstvennogo agrarnogo universiteta. 2014. № 98. S. 1-13.

2. Orlov A.I. Teorija nechetkih mnozhestv - chast' teorii verojatnostej // Politematicheskij setevoj jelektronnyj nauchnyj zhurnal Kubanskogo gosudarstvennogo agrarnogo universiteta. 2013. № 92. S. 51-60.

3. Orlov A.I. Statistika nechetkih dannyh // Politematicheskij setevoj jelektronnyj nauchnyj zhurnal Kubanskogo gosudarstvennogo agrarnogo universiteta. 2016. № 119. S. 7591.

4. Orlov A.I. Osnovnye idei statistiki interval'nyh dannyh // Politematicheskij setevoj jelektronnyj nauchnyj zhurnal Kubanskogo gosudarstvennogo agrarnogo universiteta. 2013. № 94. S. 55-70.

5. Orlov A.I., Lucenko E.V. Sistemnaja nechetkaja interval'naja matematika. Monografija (nauchnoe izdanie). - Krasnodar, KubGAU. 2014. - 600 s.

6. Orlov A.I. Asimptotika kvantovanija i vybor chisla gradacij v sociologicheskih anketah // V sb.: Matematicheskie metody i modeli v sociologii. - M.: ISI AN SSSR, 1977. S. $42-55$.

7. Orlov A.I. Ustojchivost' v social'no-jekonomicheskih modeljah. - M.: Nauka, 1979. $-296 \mathrm{~s}$.

8. Orlov A.I. Ustojchivye jekonomiko-matematicheskie metody i modeli. Saarbrücken (Germany), Lambert Academic Publishing, 2011. - 436 s.

9. Orlov A.I. Novyj podhod $k$ izucheniju ustojchivosti vyvodov $v$ matematicheskih modeljah // Politematicheskij setevoj jelektronnyj nauchnyj zhurnal Kubanskogo gosudarstvennogo agrarnogo universiteta. 2014. № 100. S. 146-176.

10. Orlov A.I. Prikladnaja statistika. - M.: Jekzamen, 2006. - 672 s.

11. Orlov A.I. Teoreticheskie instrumenty statisticheskih metodov // Politematicheskij setevoj jelektronnyj nauchnyj zhurnal Kubanskogo gosudarstvennogo agrarnogo universiteta. 2014. № 101. S. 253-274.

12. Shubkin V.N. Sociologicheskie opyty. - M.: Mysl', 1970. - 256 s.

13. Shoshin P.B. Razmytye chisla kak sredstvo opisanija sub\#ektivnyh velichin // Statisticheskie metody analiza jekspertnyh ocenok. - M.: Nauka, 1977. - S. 234-250.

14. Saganenko G.I. Nadezhnost' rezul'tatov sociologicheskogo issledovanija. - L.: Nauka, 1983. - $180 \mathrm{~s}$.

15. Shljapentoh V.Je. Problemy dostovernosti statisticheskoj informacii V sociologicheskih issledovanijah. - M.: Statistika, 1973. - 144 s.

16. Kolmogorov A.N., Fomin S.V. Jelementy teorii funkcij i funkcional'nogo analiza. Uchebnik. - M.: Nauka, 1972. - 496 s. 
17. Gnedenko B.V. Kurs teorii verojatnostej: Uchebnik. 7-e izd., ispravl. - M.: Jeditorial URSS, 2001. $320 \mathrm{~s}$.

18. Lojev M. Teorija verojatnostej. - M.: IL, 1962. - $720 \mathrm{~s}$.

19. Orlov A.I. Predel'naja teorija neparametricheskih statistik // Politematicheskij setevoj jelektronnyj nauchnyj zhurnal Kubanskogo gosudarstvennogo agrarnogo universiteta. 2014. № 100. S. 31-52.

20. Morgenshtern O. O tochnosti jekonomiko-statisticheskih nabljudenij. - M.: Statistika, 1968. - $396 \mathrm{~s}$.

21. Titma M.H., Tooding L.M. Matematicheskie metody v arsenale sociologa. / Sociologicheskie issledovanija. 1986. №4. S.123-128.

22. Glotov V.A., Pavel'eva V.V. Vektornaja stratifikacija. - M.: Nauka, 1984. - 94 S.

23. Il'jasov F.N. Jeksperimental'noe obosnovanie kolichestva delenij shkaly. I Sociologicheskie issledovanija. 1984. №4. S.113-116.

24. Litvak B.G. O vybore delenij shkaly. - V sb.: Statisticheskie metody analiza jekspertnyh ocenok. - M.: Nauka, 1977. - S.228-234.

25. Orlov A.I. Optimal'nyj plan upravlenija zapasami nel'zja najti na osnove formuly kvadratnogo kornja // Politematicheskij setevoj jelektronnyj nauchnyj zhurnal Kubanskogo gosudarstvennogo agrarnogo universiteta. 2015. № 106. S. 270-300.

26. Orlov A.I. Organizacionno-jekonomicheskoe modelirovanie : uchebnik : v 3 ch. Ch.3. Statisticheskie metody analiza dannyh. - M.: Izd-vo MGTU im. N.Je. Baumana, 2012. $-624 \mathrm{~s}$.

27. Lavrenchenko A.S. Reshenie zadach optimal'nogo upravlenija zapasami pri nestacionarnom puassonovskom potoke trebovanij. // Sb. nauchnyh trudov / Moskovskij aviacionnyj institut. - 1974. - Vyp.306. - S. 62-72.

28. Orlov A.I. Otkaz ot puassonovosti sprosa v odnoj modeli upravlenija zapasami // Issledovanija po verojatnostno-statisticheskomu modelirovaniju real'nyh sistem. - M.: Izdvo CJeMI AN SSSR, 1977. - S. 93-96.

29. Rotar' G.V. Ob odnoj zadache upravlenija rezervami // Jekonomika i matematicheskie metody. 1976. T.12. №4. S. 733-739.

30. Rotar' G.V. Odna zadacha ob upravlenii rezervami // Teorija verojatnostej i ee primenenija. 1972. T.XVII. Vyp.3. S.597-599.

31. Saul'ev V.K., Lavrenchenko A.S. Matematicheskaja teorija optimal'nogo upravlenija zapasami. - M.: MAI, 1974. - $188 \mathrm{~s}$.

32. Hedli Dzh., Uajtin T. Analiz sistem upravlenija zapasami. - M.: Nauka, 1969. $-512 \mathrm{~s}$.

33. Feller V. Vvedenie v teoriju verojatnostej i ejo prilozhenija: Tom 2. - M.: Mir, 1984. $-738 \mathrm{~s}$.

34. Voskresenskij A.V. Princip invariantnosti v odnoj modeli upravlenija zapasami / Dipl. rabota (rukovoditel' A.I. Orlov). - M.: Meh.-mat. f-t MGU im. M.V. Lomonosova, 1977.

35. Orlov A.I., Voskresenskij A.V. Princip invariantnosti v odnoj modeli upravlenija zapasami // Prikladnoj mnogomernyj statisticheskij analiz. Uchenye zapiski po statistike, t.33. - M.: Nauka, 1978. - S.307-311.

36. Orlov A.I. Gorizontnaja ustojchivost' dvuhurovnevoj modeli upravlenija zapasami // Mnogomernyj statisticheskij analiz (matematicheskoe obespechenie). - M.: Izd-vo CJeMI AN SSSR, 1979. - S.187-199.

37. Orlov A.I. Matematicheskie metody v sociologii za sorok pjat' let // Politematicheskij setevoj jelektronnyj nauchnyj zhurnal Kubanskogo gosudarstvennogo agrarnogo universiteta. 2016. № 117. S. 91-119. 